\title{
Weak Equivalence Transformations for a Class of Models in Biomathematics
}

\author{
Igor Leite Freire ${ }^{1}$ and Mariano Torrisi ${ }^{2}$ \\ ${ }^{1}$ Centro de Matemática, Computação e Cognição, Universidade Federal do ABC (UFABC), Rua Santa Adélia 166, \\ Bairro Bangu, 09.210-170 Santo André, SP, Brazil \\ ${ }^{2}$ Dipartimento di Matematica e Informatica, Università Degli Studi di Catania, Viale Andrea Doria 6, 95125 Catania, Italy
}

Correspondence should be addressed to Igor Leite Freire; igor.freire@ufabc.edu.br

Received 29 November 2013; Accepted 11 January 2014; Published 16 March 2014

Academic Editor: Maria Gandarias

Copyright (C) 2014 I. L. Freire and M. Torrisi. This is an open access article distributed under the Creative Commons Attribution License, which permits unrestricted use, distribution, and reproduction in any medium, provided the original work is properly cited.

A class of reaction-diffusion systems unifying several Aedes aegypti population dynamics models is considered. Equivalence transformations are found. Extensions of the principal Lie algebra are derived for some particular cases.

\section{Introduction}

In this paper we focus our attention on the following class of nonlinear advection-reaction-diffusion systems:

$$
\begin{gathered}
u_{t}=\left(f(u) u_{x}\right)_{x}+g\left(u, v, u_{x}\right), \quad g_{u_{x}} \neq 0, \\
v_{t}=h(u, v) .
\end{gathered}
$$

These systems can describe the evolution of the densities $u$ and $v$ of two interacting populations where the balance equation for $u$ takes into account not only the reaction-diffusion effects but also some advection effects while the balance equation for density $v$ takes into account only the so-called reaction terms. The advection effects are due to the presence in the function $g$ of the gradient $u_{x}$ and appear when the individuals of population $u$ feel external stimuli as, for instance, wind effects or water currents. Class (1) can be considered a generalization of the equations with the typical properties of the already known Aedes aegypti mathematical models [1-3]. We recall them shortly in the following.

The Aedes aegypti mosquitos are the main vector of dengue, a viral disease that causes the so-called dengue hemorrhagic fever characterized by coagulation problems often leading the infected individual to death. Since the subtropical zone climate and environmental conditions are favorable to the development of Aedes aegypti, dengue is a serious public health problem in many countries around the world. However, due to the global warming, the interest in such considered mosquitoes is not restricted to those places affected by the disease, but it is also of interest for those countries whose weather, in the next decades, can become similar to the current environmental found in the subtropical zone. Therefore, the interest in modeling such a vector is not only a theoretical deal but also a way for finding methods and alternatives to overcome and control the problems arising from the dispersal dynamics of the mosquitos and, consequently, the propagation of the disease.

The following system

$$
\begin{gathered}
u_{t}=\left(u^{p} u_{x}\right)_{x}-2 v u^{q} u_{x}+\frac{\gamma}{k} v(1-u)-m_{1} u, \\
v_{t}=k(1-v) u-\left(m_{2}+\gamma\right) v,
\end{gathered}
$$

where $p, q \in \mathbb{R}$, belongs to class (1). It was introduced in [2] as a generalization of a model studied in [1].

We recall that in (2), as well as in [1], $u$ and $v$ are, respectively, nondimensional densities of winged and aquatic populations of mosquitoes and $k, \gamma, m_{1}$, and $m_{2}$ are nondimensional, in general, positive parameters, $v \in \mathbb{R}$. Specifically $k$ is the ratio between two constants $\bar{k}_{1}$ and $\bar{k}_{2}$, which are, respectively, the carrying capacity related to the amount of findable nutrients and the carrying capacity effect dependent on the occupation of the available breeder and $\gamma$ denotes the 
specific rate of maturation of the aquatic form into winged female mosquitoes, while $m_{1}$ and $m_{2}$ are, respectively, the mortality of winged and the mortality of aquatic populations. Finally $v$ denotes a constant of velocity for flux due to wind currents that, in general, generate an advection motion of large masses of the winged population and consequently can facilitate a quick advance of the infestation. For further details, see [4] and references therein.

Another system belonging to (1) is

$$
\begin{gathered}
u_{t}=\left(u^{p} u_{x}\right)_{x}-2 v u^{q} u_{x}+\frac{\gamma}{k} v+\left(\frac{\gamma}{k}-m_{1}\right) u, \\
v_{t}=k u+\left(k-m_{2}-\gamma\right) v,
\end{gathered}
$$

which was also introduced in [2] starting from (2) and, due to the weak interaction between the aquatic and winged populations, by modifying the source terms.

The first equation of (3) gives the time rate of change of the mosquitoes density as a sum of the growth terms $(\gamma / k) v$, of the per capita death rate $\left((\gamma / k)-\mu_{1}\right) u$ and the diffusiveadvective flux due to the movement of mosquito population.

The second equation gives the corresponding time rate of change of the density of aquatic population as a sum of the growth term $k u$ of aquatic population, due to the new egg depositions of female mosquitoes, with per capita death rate $\left(k-\mu_{2}\right) v$ of aquatic population. The term $-\gamma v$ represents the loss due to the change of the aquatic into winged form; see [4].

The family of system (1) contains arbitrary functions or numerical parameters, which specifies the individual characteristics of phenomena belonging to large subclasses. In this sense, the knowledge of equivalence transformations can provide us with certain relations between the solutions of different phenomena of the same class and allows us to get symmetries in a quite direct way.

Following [5], an equivalence transformation is a nondegenerated change of independent and dependent variables $t$, $x, u$, and $v$ into $\widehat{t}, \widehat{x}, \widehat{u}$, and $\widehat{v}$ :

$$
\begin{aligned}
& x=x(\widehat{x}, \widehat{t}, \widehat{u}, \widehat{v}), \\
& t=t(\widehat{x}, \widehat{t}, \widehat{u}, \widehat{v}), \\
& u=u(\widehat{x}, \widehat{t}, \widehat{u}, \widehat{v}), \\
& v=v(\widehat{x}, \widehat{t}, \widehat{u}, \widehat{v}),
\end{aligned}
$$

which maps a system of class (1) in another one of the same class, that is, in an equation preserving differential structure but, in general, with

$$
\begin{gathered}
\widehat{f}(\widehat{u}) \neq f(u), \quad \widehat{g}\left(\widehat{u}, \widehat{v}, \widehat{u}_{\widehat{x}}\right) \neq g\left(u, v, u_{x}\right), \\
\widehat{h}(\widehat{u}, \widehat{v}) \neq h(u, v) .
\end{gathered}
$$

Of course in the case

$$
\begin{gathered}
\widehat{f}(\widehat{u})=f(u), \quad \widehat{g}\left(\widehat{u}, \widehat{v}, \widehat{u}_{\widehat{x}}\right)=g\left(u, v, u_{x}\right), \\
\widehat{h}(\widehat{u}, \widehat{v})=h(u, v)
\end{gathered}
$$

an equivalence transformation becomes a symmetry.
In this paper we look for certain equivalence transformations for the class of systems (1) in order to find symmetries for special systems belonging to (1) and to get information about constitutive parameters $f, g$, and $h$ appearing there. Moreover we wish to stress that, as it is known, an equivalence transformation maps solutions of an equation in solutions of the transformed equation [6]. Then in order to find solutions for a certain equation one can look for the equivalence transformations that bring the equation in simpler other ones whose solutions are well studied; see, for example, $[7,8]$ and references inside.

The plan of the paper is as follows. In the next section we provide some elements about equivalence transformations. In Section 3 we apply these concepts in order to obtain a set of weak equivalence generators. In Section 4, after having introduced a projection theorem, we show how to apply it to find symmetries of (1). In Section 5, after having introduced a special structure of the advection-reaction function $g$ that generalizes that one used in (3), we find extensions with respect to the principal Lie algebra. Conclusions and final remarks are given in Section 6.

\section{Elements on Equivalence Transformations}

In the past differential equation literature it is possible to find several examples of equivalence transformations. The direct search for the most general equivalence transformations through the finite form of the transformation is connected with considerable computational difficulties and quite often leads to partial solutions of the problem (e.g., $[9,10])$.

A systematic treatment to look for continuous equivalence transformations by using the Lie infinitesimal criterion was suggested by Ovsiannikov [11].

In general, the equivalence transformations for class (1) can be considered as transformations acting on point of the basic augmented space

$$
A \equiv\left\{t, x, u, v, u_{t}, u_{x}, v_{t}, v_{x}, f, g, h\right\} .
$$

The previous elements allow us to consider, in the following, the one-parameter equivalence transformations as a group of transformations, acting on the basic augmented space $A$, of the type

$$
\begin{aligned}
& x=x(\widehat{x}, \widehat{t}, \widehat{u}, \widehat{v}, \varepsilon), \\
& t=t(\widehat{x}, \widehat{t}, \widehat{u}, \widehat{v}, \varepsilon), \\
& u=u(\widehat{x}, \widehat{t}, \widehat{u}, \widehat{v}, \varepsilon), \\
& v=v(\widehat{x}, \widehat{t}, \widehat{u}, \widehat{v}, \varepsilon),
\end{aligned}
$$

$$
\begin{aligned}
& f=f\left(\widehat{x}, \widehat{t}, \widehat{u}, \widehat{v}, \widehat{u}_{\hat{t}}, \widehat{u}_{\hat{x}}, \widehat{v}_{\hat{t}}, \widehat{v}_{\widehat{x}}, \widehat{f}, \widehat{g}, \widehat{h}, \varepsilon\right), \\
& g=g\left(\widehat{x}, \widehat{t}, \widehat{u}, \widehat{v}, \widehat{u}_{\hat{t}}, \widehat{u}_{\widehat{x}}, \widehat{v}_{\hat{t}}, \widehat{v}_{\widehat{x}}, \widehat{f}, \widehat{g}, \widehat{h}, \varepsilon\right), \\
& h=h\left(\widehat{x}, \widehat{t}, \widehat{u}, \widehat{v}, \widehat{u}_{\hat{t}}, \widehat{u}_{\widehat{x}}, \widehat{v}_{\hat{t}}, \widehat{v}_{\hat{x}}, \widehat{f}, \widehat{g}, \widehat{h}, \varepsilon\right),
\end{aligned}
$$

which is locally a $C^{\infty}$-diffeomorphism, depending analytically on the parameter $\varepsilon$ in a neighborhood of $\varepsilon=0$, and reduces to the identity transformation for $\varepsilon=0$. 
Following [6, 11-14] (see also, e.g., [5, 15-17] ) we consider the infinitesimal generator of the equivalence transformations (8) of the systems (1) that reads as follows:

$$
Y=\xi^{1} \partial_{x}+\xi^{2} \partial_{t}+\eta^{1} \partial_{u}+\eta^{2} \partial_{v}+\mu^{1} \partial_{f}+\mu^{2} \partial_{g}+\mu^{3} \partial_{h},
$$

where the infinitesimal components $\xi^{1}, \xi^{2}, \eta^{1}$, and $\eta^{2}$ are sought depending on $x, t, u$, and $v$, while the infinitesimal components $\mu^{i}(i=1,2,3)$ are sought, at least in principle, depending on $x, t, u, v, u_{t}, u_{x}, u_{x}, v_{t}, v_{x}, f, g$, and $h$. In order to obtain the determining system which allows us to get the infinitesimal coordinates $\xi^{i}, \eta^{i}$, and $\mu^{j}(i=1,2$ and $j=$ $1,2,3)$, we apply the Lie-Ovsiannikov infinitesimal criterion by requiring the invariance, with respect to suitable prolongations $Y^{(1)}$ and $Y^{(2)}$ of (9), of the following equations:

$$
\begin{gathered}
u_{t}-\left(f u_{x}\right)_{x}-g=0, \\
v_{t}-h=0,
\end{gathered}
$$

together with the invariance of the auxiliary conditions $[13,14$, 18, 19]:

$$
\begin{aligned}
f_{t} & =f_{x}=f_{v}=f_{u_{x}}=f_{u_{t}}=f_{v_{x}}=f_{v_{t}} \\
& =g_{t}=g_{x}=g_{u_{t}}=g_{v_{t}}=g_{v_{x}}=0, \\
h_{t} & =h_{x}=h_{u_{x}}=h_{u_{t}}=h_{v_{x}}=h_{v_{t}}=0,
\end{aligned}
$$

where $u$ and $v$ are $(t, x)$ functions while $f, g$, and $h$ are considered as functions depending, a priori, on $(t, x, u$, $\left.v, u_{t}, u_{x}, v_{t}, v_{x}\right)$. All of these functions are assumed to be analytical. The constraints, given by (11), characterize the functional dependence of $f, g$, and $h$.

In this paper, instead, in view of further applications and following [20], we modify the previous classical procedure by looking for equivalence transformations whose generators are got by solving the determining system obtained from the following invariance conditions:

$$
\begin{aligned}
& \left.Y^{(2)}\left[u_{t}-\left(f(u) u_{x}\right)_{x}-g\left(u, v, u_{x}\right)\right]\right|_{\substack{u_{t}-\left(f(u) u_{x}\right)_{x}-g\left(u, v, u_{x}\right)=0 \\
v_{t}-h(u, v)=0}} \\
& =0 \\
& \left.\quad Y^{(1)}\left[v_{t}-h(u, v)\right]\right|_{\substack{u_{t}-\left(f(u) u_{x}\right)_{x}-g\left(u, v, u_{x}\right)=0, v_{t}-h(u, v)=0}}=0
\end{aligned}
$$

As the functional dependences of the parameters $f, g$, and $h$ are known a priori we do not require the invariance of the auxiliary conditions (11). In this way we work in a basic augmented space $A \equiv\left\{t, x, u, v, u_{x}, f, g, h\right\}$. Therefore the $\mu^{i}$ components must be sought, at least in principle, depending on $x, t, u, v, u_{x}, f, g$, and $h$.

The infinitesimal operators, obtained by following this shortening procedure, can generate transformations that map equations of our class into new equations of the same class where the transformed arbitrary functions may have new additional functional dependencies. Such transformations are called weak equivalence transformations $[13,14]$.
With respect to the application in biomathematical models, equivalence and weak equivalence transformations were applied not only to study of tumor models [21, 22] but also to the population dynamics in $[20,23,24]$.

\section{Calculation of Weak Equivalence Transformations}

In order to avoid long formulas and write $Y^{(1)}$ and $Y^{(2)}$ in a compact way, we put

$$
\begin{gathered}
x=x^{1}, \quad t=x^{2}, \\
u=u^{1}, \quad v=u^{2}, \\
f=h^{1}, \quad g=h^{2}, \quad h=h^{3} .
\end{gathered}
$$

For this reason system (1) is rewritten as

$$
\begin{gathered}
u_{x^{2}}^{1}-h^{2}-h_{u^{1}}^{1}\left(u_{x^{1}}^{1}\right)^{2}-h^{1} u_{x^{1} x^{1}}^{1}=0 \\
u_{x^{2}}^{2}-h^{3}=0
\end{gathered}
$$

while the equivalence generator assumes the following form:

$$
Y=\xi^{i} \partial_{x^{i}}+\eta^{\alpha} \partial_{u^{\alpha}}+\mu^{A}, \partial_{h^{A}},
$$

where $i=1,2, \alpha=1,2$, and $A=1,2,3$. Here the summation over the repeated indices is presupposed.

After putting

$$
\begin{gathered}
\left(z^{1}, z^{2}, z^{3}, z^{4}, z^{5}\right)=\left(x^{1}, x^{2}, u^{1}, u^{2}, u_{1}^{1}\right), \\
\left(v^{1}, v^{2}, v^{3}, v^{4}, v^{5}\right)=\left(\xi^{1}, \xi^{2}, \eta^{1}, \eta^{2}, \zeta_{1}^{1}\right), \\
u_{i}^{\alpha}=u_{x^{i}}^{\alpha}, \quad h_{a}^{A}=h_{z^{a}}^{A}, \\
\widetilde{D}_{a}^{e}=\partial_{z^{a}}+h_{a}^{A} \partial_{h^{A}}, \\
D_{j}^{e}=\partial_{x^{j}}+u_{j}^{\alpha} \partial_{u^{\alpha}}+u_{i j}^{\alpha} \partial_{u_{i}^{\alpha}}+\cdots,
\end{gathered}
$$

the prolongations $Y^{(1)}$ and $Y^{(2)}$ assume the following form:

$$
\begin{gathered}
Y^{(1)}=Y+\zeta_{j}^{\alpha} \partial_{u_{j}^{\alpha}}+\omega_{a}^{A} \partial_{h_{a}^{A}}, \\
Y^{(2)}=Y^{(1)}+\zeta_{11}^{1} \partial_{u_{11}^{1}}=Y+\zeta_{j}^{\alpha} \partial_{u_{j}^{\alpha}}+\omega_{a}^{A} \partial_{h_{a}^{A}}+\zeta_{11}^{1} \partial_{u_{11}^{1}},
\end{gathered}
$$

where

$$
\begin{aligned}
\zeta_{j}^{\alpha} & =D_{j}^{e} \eta^{\alpha}-u_{k}^{\alpha} D_{j}^{e} \xi^{k}, \\
\zeta_{i j}^{\alpha} & =D_{j}^{e} \zeta_{j}^{\alpha}-u_{i k}^{\alpha} D_{j}^{e} \xi^{k}, \\
\omega_{a}^{A} & =\widetilde{D}_{a}^{e} \mu^{A}-h_{b}^{A} \widetilde{D}_{a}^{e} \nu^{b} .
\end{aligned}
$$

The invariant conditions read

$$
\begin{gathered}
Y^{(2)} F_{1}=\zeta_{2}^{1}-\mu^{2}-2 \zeta_{1}^{1} h_{u^{1}}^{1} u_{1}^{1}-\left(\omega_{3}^{1}\right)\left(u_{1}^{1}\right)^{2} \\
-\left(\mu^{1}\right) u_{11}^{1}-h^{1} \zeta_{11}^{1}=0 \\
Y^{(1)} F_{2}=\zeta_{2}^{2}-\mu^{3}=0,
\end{gathered}
$$


both under the constraints (15), which are after (18)

$$
\begin{gathered}
u_{2}^{1}=h^{2}+h_{u^{1}}^{1}\left(u_{1}^{1}\right)^{2}+h^{1} u_{11}^{1}, \\
u_{2}^{2}=h^{3} .
\end{gathered}
$$
by

The coefficients $\zeta_{1}^{1}, \zeta_{2}^{1}, \zeta_{2}^{2}$, and $\omega_{3}^{1}$ are given, respectively,

$$
\begin{aligned}
\zeta_{1}^{1}= & \eta_{1}^{1}+\left(\eta_{u^{1}}^{1}-\xi_{1}^{1}\right) u_{1}^{1}-\xi_{1}^{2} u_{2}^{1}+\eta_{u^{2}}^{1} u_{1}^{2} \\
& -\xi_{u^{1}}^{1}\left(u_{1}^{1}\right)^{2}-\xi_{u^{2}}^{1} u_{1}^{1} u_{1}^{2}-\xi_{u^{1}}^{2} u_{1}^{1} u_{2}^{1}-\xi_{u^{2}}^{2} u_{2}^{1} u_{1}^{2}, \\
\zeta_{2}^{1}= & \eta_{2}^{1}-\xi_{2}^{1} u_{1}^{1}+\left(\eta_{u^{1}}^{1}-\xi_{2}^{2}\right) u_{2}^{1}+\eta_{u^{2}}^{1} u_{2}^{2} \\
& -\xi_{u^{1}}^{1} u_{1}^{1} u_{2}^{1}-\xi_{u^{2}}^{1} u_{1}^{1} u_{2}^{2}-\xi_{u^{1}}^{2}\left(u_{2}^{1}\right)^{2}-\xi_{u^{2}}^{2} u_{2}^{1} u_{2}^{2}, \\
\zeta_{2}^{2}= & \eta_{2}^{2}+\eta_{u^{1}}^{2} u_{2}^{1}+\left(\eta_{u^{2}}^{2}-\xi_{2}^{2}\right) u_{2}^{2}-\xi_{2}^{1} u_{1}^{2} \\
& -\xi_{u^{1}}^{1} u_{1}^{2} u_{2}^{1}-\xi_{u^{2}}^{1} u_{1}^{2} u_{2}^{2}-\xi_{u^{1}}^{2} u_{2}^{1} u_{2}^{2}-\xi_{u^{2}}^{2}\left(u_{2}^{2}\right)^{2}, \\
\omega_{3}^{1}= & \mu_{u^{1}}^{1}+h_{u^{1}}^{1} \mu_{h^{1}}^{1}+h_{u^{1}}^{2} \mu_{h^{2}}^{1}+h_{u^{1}}^{3} \mu_{h^{3}}^{1}-h_{u^{1}}^{1} \eta_{u^{1}}^{1} .
\end{aligned}
$$

Taking into account (24) and (27), we can write

$$
\begin{aligned}
Y^{(1)} F_{2}= & \eta_{2}^{2}+\eta_{u^{1}}^{2} u_{2}^{1}+\left(\eta_{u^{2}}^{2}-\xi_{2}^{2}\right) u_{2}^{2}-\xi_{2}^{1} u_{1}^{2}-\xi_{u^{1}}^{1} u_{1}^{2} u_{2}^{1} \\
& -\xi_{u^{2}}^{1} u_{1}^{2} u_{2}^{2}-\xi_{u^{1}}^{2} u_{2}^{1} u_{2}^{2}-\xi_{u^{2}}^{2}\left(u_{2}^{2}\right)^{2}-\mu^{3}=0
\end{aligned}
$$

with the constraints (25) and (26).

Then, substituting (25) and (26) into (28), we get

$$
\begin{aligned}
\eta_{2}^{2}+ & \eta_{u^{1}}^{2}\left(h^{2}+h_{u^{1}}^{1}\left(u_{1}^{1}\right)^{2}+h^{1} u_{11}^{1}\right)+\left(\eta_{u^{2}}^{2}-\xi_{2}^{2}\right) h^{3} \\
& -\xi_{2}^{1} u_{1}^{2}-\xi_{u^{1}}^{1} u_{1}^{2}\left(h^{2}+h_{u^{1}}^{1}\left(u_{1}^{1}\right)^{2}+h^{1} u_{11}^{1}\right) \\
& -\xi_{u^{2}}^{1} u_{1}^{2} h^{3}-\xi_{u^{1}}^{2}\left(h^{2}+h_{u^{1}}^{1}\left(u_{1}^{1}\right)^{2}+h^{1} u_{11}^{1}\right) h^{3} \\
& -\xi_{u^{2}}^{2}\left(h^{3}\right)^{2}-\mu^{3}=0 .
\end{aligned}
$$

From this condition we obtain the following determining equations:

$$
\begin{gathered}
\eta_{2}^{2}+\left(\eta_{u^{2}}^{2}-\xi_{2}^{2}\right) h^{3}-\xi_{u^{2}}^{2}\left(h^{3}\right)^{2}-\mu^{3}=0, \\
\xi_{2}^{1}=0, \quad \xi_{u^{1}}^{1}=0, \quad \xi_{u^{2}}^{1}=0, \\
\eta_{u^{1}}^{2}=0, \quad \xi_{u^{1}}^{2}=0 .
\end{gathered}
$$

Then it follows that

$$
\begin{aligned}
& \xi^{1}=\xi^{1}\left(x^{1}\right), \quad \xi^{2}=\xi^{2}\left(x^{1}, x^{2}, u^{2}\right), \quad \eta^{2}=\eta^{2}\left(x^{1}, x^{2}, u^{2}\right), \\
& \eta^{1}=\eta^{1}\left(x^{1}, x^{2}, u^{1}, u^{2}\right), \quad \mu^{3}=\eta_{2}^{2}+\left(\eta_{u^{2}}^{2}-\xi_{2}^{2}\right) h^{3}-\xi_{u^{2}}^{2}\left(h^{3}\right)^{2}
\end{aligned}
$$

and, consequently, $\mu_{u_{1}^{1}}^{3}=0$.
Following the same procedure we can write the invariance condition (23) as

$$
\begin{aligned}
Y^{(2)} F_{1}= & \eta_{2}^{1}+\left(\eta_{u^{1}}^{1}-\xi_{2}^{2}\right)\left(h^{2}+h_{u^{1}}^{1}\left(u_{1}^{1}\right)^{2}+h^{1} u_{11}^{1}\right) \\
& +\eta_{u^{2}}^{1} h^{3}-\xi_{u^{2}}^{2}\left(h^{2}+h_{u^{1}}^{1}\left(u_{1}^{1}\right)^{2}+h^{1} u_{11}^{1}\right) h^{3} \\
& -\mu^{2}-2\left\{\eta_{1}^{1}+\left(\eta_{u^{1}}^{1}-\xi_{1}^{1}\right) u_{1}^{1}\right. \\
& -\xi_{1}^{2}\left(h^{2}+h_{u^{1}}^{1}\left(u_{1}^{1}\right)^{2}+h^{1} u_{11}^{1}\right)+\eta_{u^{2}}^{1} u_{1}^{2}+ \\
& -\left(\mu_{u^{1}}^{1}+h_{u^{2}}^{1} \mu_{h^{1}}^{1}+h_{u^{1}}^{2} \mu_{h^{2}}^{1}+h_{u^{1}}^{3} \mu_{h^{3}}^{1}-h_{u^{1}}^{1} \eta_{u^{1}}^{1}\right) \\
& \times\left(u_{1}^{1}\right)^{2}-\left(\mu^{1}\right) u_{11}^{1} \\
& -h^{1}\left(D_{1}^{e} \eta_{1}^{1}+D_{1}^{e}\left(\eta_{u^{1}}^{1}-\xi_{1}^{1}\right) u_{1}^{1}+\left(\eta_{u^{1}}^{1}-\xi_{1}^{1}\right) u_{11}^{1}\right. \\
& \left.+\left(D_{1}^{e} \eta_{u^{2}}^{1}\right) u_{1}^{2}+\eta_{u^{2}}^{1} u_{11}^{2}\right) \\
& -h_{u^{1}}^{1} u_{1}^{1} \\
& -D_{1}^{e}\left(\xi_{1}^{2}\right)\left(h^{2}+h_{u^{1}}^{1}\left(u_{1}^{1}\right)^{2}+h^{1} u_{11}^{1}\right) \\
& -\xi_{1}^{2} u_{21}^{1}-D_{1}^{e} \xi_{u^{2}}^{2}\left(h^{2}+h_{u^{1}}^{1}\left(u_{1}^{1}\right)^{2}+h^{1} u_{11}^{1}\right) u_{1}^{2} \\
& -\xi_{u^{2}}^{2} u_{21}^{1} u_{1}^{2}-\xi_{u^{2}}^{2}\left(h^{2}+h_{u^{1}}^{1}\left(u_{1}^{1}\right)^{2}+h^{1} u_{11}^{1}\right) u_{11}^{2} \\
& -0 .
\end{aligned}
$$

Collecting the terms with $\left(h_{u^{1}}^{1}\right)^{2}$ and with $h_{u^{1}}^{1}$ and equating their respective coefficients to zero it is obtained that,

$$
\begin{gathered}
\xi_{1}^{2}=0, \quad \xi_{u^{2}}^{2}=0, \quad \eta_{1}^{1}=0, \\
\mu_{h^{1}}^{1}=2 \xi_{1}^{1}-\xi_{2}^{2}, \quad \eta_{u^{2}}^{1}=0 \quad \eta_{1}^{1}=0 .
\end{gathered}
$$

Thus, from the coefficients of $h_{u^{1}}^{2}$ and $h_{u^{1}}^{3}$

$$
\mu_{h^{2}}^{1}=\mu_{h^{3}}^{1}=0 .
$$

Considering the coefficients of $u_{11}^{1}$ we get

$$
\mu^{1}=\left(2 \xi_{1}^{1}-\xi_{2}^{2}\right) h^{1}=0 \Longrightarrow \mu_{u^{1}}^{1}=0 .
$$

The remaining terms of (32) give the following form for the infinitesimal component of $h^{2}$ :

$$
\mu^{2}=\eta_{2}^{1}+\left(\eta_{u^{1}}^{1}-\xi_{2}^{2}\right) h^{2}+\left(\xi_{11}^{1}-\eta_{u^{1} u^{1}}^{1} u_{1}^{1}\right) h^{1} .
$$

Therefore, once having taken into account all restrictions obtained we are finally able to write the following infinitesimal components for the weak equivalence generators:

$$
\begin{gathered}
\xi^{1}=\alpha\left(x^{1}\right), \quad \xi^{2}=\beta\left(x^{2}\right), \\
\eta^{1}=\delta\left(x^{2}, u^{1}\right), \quad \eta^{2}=\lambda\left(x^{1}, x^{2}, u^{2}\right), \\
\mu^{1}=\left(2 \alpha^{\prime}-\beta^{\prime}\right) h^{1}, \quad \mu^{3}=\left(\lambda_{u^{2}}-\beta^{\prime}\right) h^{3}+\lambda_{x^{2}},
\end{gathered}
$$


while, from (36), we get

$$
\mu^{2}=\delta_{x^{2}}+\left(\delta_{u^{1}}-\beta_{x^{2}}\right) h^{2}+\left(\alpha_{x^{1} x^{1}}-\delta_{u^{1} u^{1}} u_{x^{1}}^{1}\right) h^{1}
$$

where $\alpha\left(x^{1}\right), \beta\left(x^{2}\right), \delta\left(x^{2}, u^{1}\right)$, and $\lambda\left(x^{1}, x^{2}, u^{2}\right)$ are arbitrary real functions of their arguments. Then, going back to the original variables, the most general operator of these continuous weak equivalence transformations reads

$$
\begin{aligned}
Y= & \alpha(x) \partial_{x}+\beta(t) \partial_{t}+\delta(t, u) \partial_{u} \\
& +\lambda(x, t, v) \partial_{v}+\left(2 \alpha^{\prime}-\beta^{\prime}\right) f \partial_{f} \\
& +\left(\delta_{t}+\left(\delta_{u}-\beta_{t}\right) g+\left(\alpha^{\prime \prime}-\delta_{u u} u_{x}\right) f\right) \partial_{g} \\
& +\left(\left(\lambda_{v}-\beta^{\prime}\right) h+\lambda_{t}\right) \partial_{h} .
\end{aligned}
$$

\section{Symmetries for the System (1)}

In the next sections in order to carry out symmetries for the system (1) we do not use the classical Lie approach. Instead of the mentioned method we apply the projection theorem, introduced in [25] and eventually reconsidered in [13, 14, 18, 19]. In agreement with these references, we can affirm the following.

Theorem 1. Let

$$
\begin{aligned}
Y= & \alpha(x) \partial_{x}+\beta(t) \partial_{t}+\delta(t, u) \partial_{u} \\
& +\lambda(x, t, v) \partial_{v}+\left(2 \alpha^{\prime}-\beta^{\prime}\right) f \partial_{f} \\
& +\left(\delta_{t}+\left(\delta_{u}-\beta_{t}\right) g+\left(\alpha^{\prime \prime}-\delta_{u u} u_{x}\right) f\right) \partial_{g} \\
& +\left(\left(\lambda_{v}-\beta^{\prime}\right) h+\lambda_{t}\right) \partial_{h}
\end{aligned}
$$

be an infinitesimal equivalence generator for the system (1); then the operator

$$
X=\alpha(x) \partial_{x}+\beta(t) \partial_{t}+\delta(t, u) \partial_{u}+\lambda(x, t, v) \partial_{v},
$$

which corresponds to the projection of $Y$ on the space $(x, t, u, v)$, is an infinitesimal symmetry generator of the system (1) if and only if the constitutive equations, specifying the forms of $f, h$, and $g$, are invariant with respect to $Y$.

For the system under consideration, in general, the constitutive equations whose invariance must be requested are

$$
\begin{gathered}
f=D(u), \\
g=G\left(u, v, u_{x}\right), \\
h=F(u, v) .
\end{gathered}
$$

The request of invariance

$$
\begin{aligned}
& \left.Y(f-D(u))\right|_{(42)}=0,\left.\quad Y^{(1)}\left(g-G\left(u, v, u_{x}\right)\right)\right|_{(42)}=0, \\
& \left.Y(h-F(u, v))\right|_{(42)}=0
\end{aligned}
$$

brings us to the following equations:

$$
\begin{gathered}
\mu^{1}-\eta^{1} D_{u}=0, \\
\mu^{2}-\zeta_{1}^{1} G_{u_{x}}-\eta^{1} G_{u}-\eta^{2} G_{v}=0, \\
\mu^{3}-\eta^{1} F_{u}-\eta^{2} F_{v}=0,
\end{gathered}
$$

under the restrictions (42).

Then substituting

$$
\zeta_{1}^{1}=\left(\eta_{u^{1}}^{1}-\xi_{1}^{1}\right) u_{1}^{1}=\left(\delta_{u}-\alpha^{\prime}\right) u_{x}
$$

and taking into account the constraints we can write (44) as

$$
\begin{gathered}
\left(2 \alpha^{\prime}-\beta^{\prime}\right) D(u)-\delta(t, u) D_{u}=0 \\
\delta_{t}+\left(\delta_{u}-\beta_{t}\right) G-\left(\delta_{u u} u_{x}^{2}-\alpha_{x x} u_{x}\right) D-\left(\delta_{u}-\alpha^{\prime}\right) u_{x} G_{u_{x}} \\
-\delta G_{u}-\lambda(t, x, v) G_{v}=0 \\
\left(\left(\lambda_{v}-\beta^{\prime}\right) F+\lambda_{t}\right)-\delta F_{u}-\lambda(t, x, v) F_{v}=0
\end{gathered}
$$

We recall here that the principal Lie algebra $L_{\mathscr{P}}[5,12]$ is the Lie algebra of the principal Lie group, that is, the group of the all Lie point symmetries

$$
\begin{aligned}
X= & \xi(x, t, u, v) \frac{\partial}{\partial x}+\tau(x, t, u, v) \frac{\partial}{\partial t} \\
& +\eta^{1}(x, t, u, v) \frac{\partial}{\partial u}+\eta^{2}(x, t, u, v) \frac{\partial}{\partial v}
\end{aligned}
$$

that leave the system (1) invariant for any form of the functions $D(u), G\left(u, v, u_{x}\right)$, and $F(u, v)$. In other words we can remark that the principal Lie algebra is the subalgebra of the equivalence algebra such that any operator $Y$ of this subalgebra leaves the equations $f=D(u), g=G\left(u, v, u_{x}\right)$, and $h=$ $F(u, v)$ invariant for any form of the functions $D(u)$, $G\left(u, v, u_{x}\right)$, and $F(u, v)$. Then we can say [5] the following.

Corollary 2. An equivalence operator for the system (1) belongs to the principal Lie algebra $L_{\mathscr{P}}$ if and only if $\eta^{i}=0, \mu^{j}=0$, $i=1,2$, and $j=1,2,3$.

Taking Corollary 2 into account from the previous equations (46)-(48) it is a simple matter to ascertain that the $L_{\mathscr{P}}$ $[5,12]$ is spanned by the following translation generators:

$$
X_{0}=\partial_{t}, \quad X_{1}=\partial_{x}
$$

\section{Some Extensions of $L_{\mathscr{P}}$}

In order to show some extensions of the principal algebra, which could be of interest in biomathematics, we assume that the advection-reaction function is of the form

$$
G=\rho u^{r} u_{x}^{s}+\Gamma_{1} u^{a}+\Gamma_{2} v^{b}
$$

where the parameters $\rho, \Gamma_{1}, \Gamma_{2}, r, s, a$, and $b$ are constitutive parameters of the considered phenomena. 
This form of $G$ is a generalization of

$$
G=-2 v u^{q} u_{x}+\frac{\gamma}{k} v+\left(\frac{\gamma}{k}-m_{1}\right) u
$$

appearing in (3), where

$$
\rho=-2 \nu, \quad \Gamma_{1}=\left(\frac{\gamma}{k}-m_{1}\right), \quad \Gamma_{2}=\frac{\gamma}{k}
$$

Consequently in (51) we must consider $\Gamma_{2}>0$ and as limit cases $\Gamma_{1}=0$ and $a=0$. Moreover, in this section, we assume that the value $s=0$ will not be considered because in this case the advective effects disappear. We also assume that $b \neq 0$. This last restriction implies that the balance equation of the density $u$ depends on the density $v$. Finally for the sake of simplicity we omit the limit case $\Gamma_{1}=0$ and assume that the diffusion is only nonlinear; that is, $D_{u} \neq 0$.

In the following we continue the discussion of invariance conditions written in the previous section.

From (46), by deriving with respect to $x$, we get

$$
\alpha^{\prime \prime}=0 \text {. }
$$

Then

$$
\alpha=\alpha_{1} x+\alpha_{0}
$$

with $\alpha_{1}$ and $\alpha_{0}$ arbitrary constants, so (46) becomes

$$
\left(2 \alpha_{1}-\beta^{\prime}\right) D(u)-\delta(t, u) D_{u}=0
$$

while, after having taken (51) into account, (47) reads

$$
\begin{aligned}
\delta_{t} & +\left(\delta_{u}-\beta_{t}\right)\left(\rho u^{r} u_{x}^{s}+\Gamma_{1} u^{a}+\Gamma_{2} v^{b}\right) \\
& -D \delta_{u u} u_{x}^{2}-\left(\delta_{u}-\alpha_{1}\right) \rho s u^{r} u_{x}^{s}+ \\
& -\delta\left(\rho r u^{r-1} u_{x}^{s}+\Gamma_{1} a u^{a-1}\right)-\lambda(t, x, v) \Gamma_{2} b v^{b-1}=0 .
\end{aligned}
$$

From (57) we get immediately

$$
\lambda=\lambda(t, v) .
$$

In the following we analyze separately the case $s \neq 2$ and the case $s=2$.

5.1. $s \neq 2$. From $u_{x}^{2}$ coefficient we get $\delta_{u u}=0$; that is,

$$
\delta=\delta_{1}(t) u+\delta_{0}(t) .
$$

Therefore, from the remaining terms we have

$$
\begin{aligned}
\rho u^{r-1} & u_{x}^{s}\left[u\left((1-s-r) \delta_{1}+s \alpha_{1}-\beta^{\prime}\right)-r \delta_{0}\right] \\
& +\delta_{0}^{\prime}+\delta_{1}^{\prime} u+\Gamma_{1}\left((1-a) \delta_{1}-\beta^{\prime}\right)+ \\
& -a \Gamma_{1} \delta_{0} u^{a-1}+\Gamma_{2}\left(\delta_{1}-\beta^{\prime}\right) v^{b}-\Gamma_{2} \lambda b v^{b-1}=0 .
\end{aligned}
$$

As we assumed $s \neq 0$, from the coefficient of $u_{x}^{s}$ in (60) we conclude that $\delta_{0}(t)=0$ and

$$
\beta^{\prime}=(1-s-r) \delta_{1}+s \alpha_{1}
$$

Then, still from (60) we have the following constraints to consider:

$$
\begin{gathered}
\delta_{1}^{\prime} u+\Gamma_{1} u^{a}\left((1-a) \delta_{1}-\beta^{\prime}\right)=0, \\
\left(\delta_{1}-\beta^{\prime}\right) v^{b}-\lambda b v^{b-1} .
\end{gathered}
$$

From (62) two cases are obtained.

(i) Case $a \neq 1$. Then, from (62) we conclude that $\delta_{1}=$ const and it follows that

$$
\beta^{\prime}=(1-a) \delta_{1}
$$

From (64) and (61) we obtain

$$
\begin{gathered}
\alpha_{1}=\frac{s+r-a}{s} \delta_{1}, \\
\beta=(1-a) \delta_{1} t+\beta_{0},
\end{gathered}
$$

with $\beta_{0}$ and $\delta_{1}$ arbitrary constants.

The analysis of (63) leads to the following two subcases.

(1) Consider $\lambda(v)=\lambda_{0} v$, with

$$
\lambda_{0}=\frac{a}{b} \delta_{1}
$$

Taking into account the previous results and going back to (56) and (48) we get that the system (3) with $G$ of the form (51) admits the 3-dimensional Lie algebra spanned by the translations in space and time and by the following additional generator:

$$
X_{3}=(1-a) \partial_{t}+\frac{1}{s}(s+r-a) x \partial_{x}+u \partial_{u}+\frac{a}{b} v \partial_{v}
$$

provided that $D$ and $F$ are solutions of the following differential equations:

$$
\begin{gathered}
u D_{u}=\left((1+a)+2 \frac{r-a}{s}\right) D, \\
b u F_{u}+a v F_{v}=(a-b(1-a)) F .
\end{gathered}
$$

(2) Consider $\lambda(v)=\delta_{1}=0$. In this case the only symmetries admitted are translations of the independent variables and the form of $D$ and $F$ is arbitrary, so there is not an extension of the principal Lie algebra.

(ii) Case $a=1$. In this case from (62) it follows that

$$
\delta_{1}^{\prime}-\Gamma_{1} \beta^{\prime}=0
$$

After having substituted (69) into (61) we obtain

$$
\begin{gathered}
\beta(t)=-\beta_{0}+c_{1} e^{(1-s-r) \Gamma_{1} t}, \\
\delta_{1}(t)=\frac{s}{s+r-1} \alpha_{1}+c_{1} \Gamma_{1} e^{(1-s-r) \Gamma_{1} t},
\end{gathered}
$$


with $\alpha_{1}, \beta_{0}$, and $c_{1}$ arbitrary constants and once assumed $s+r-$ $1 \neq 0$. Finally we analyze the contribution of (63) from where the following two subcases arise.

(1) Consider $\lambda(t, v)=\lambda_{0}(t) v$, with

$$
\lambda_{0}(t)=\frac{1}{b} \delta_{1}(t)
$$

Then taking into account the previous results it reads

$$
\lambda_{0}(t)=\frac{1}{b}\left[\frac{s}{s+r-1} \alpha_{1}+c_{1} \Gamma_{1} e^{(1-s-r) \Gamma_{1} t}\right] .
$$

Going to put the previous result in the condition (46) and by separating the variable we get

$$
\begin{gathered}
\beta(t)=-\beta_{0}, \\
\delta_{1}(t)=\frac{s}{s+r-1} \alpha_{1}, \\
\lambda_{0}(t)=\frac{1}{b}\left[\frac{s}{s+r-1} \alpha_{1}\right],
\end{gathered}
$$

with $\alpha_{1}$ and $\beta_{0}$ arbitrary constants and provided that the diffusion coefficient $D$ is solution of

$$
u D_{u}=2\left(1+\frac{r-1}{s}\right) D
$$

From condition (48) we do not get further restrictions on the infinitesimal components of the symmetry generator but only the following constraint on the reaction function $F$ :

$$
b u F_{u}+v F_{v}=F
$$

Taking into account the arbitrariness of $\alpha_{0}$ in this case we have got a 3-dimensional Lie algebra. The additional generator is

$$
X_{3}=x \partial_{x}+\frac{s}{s+r-1} u \partial_{u}+\frac{1}{b} \frac{s}{s+r-1} v \partial_{v}
$$

In particular, from (75), setting $F=k u+\left(k-m_{2}-\gamma\right) v$, we obtain $b=1$, while from (75) we obtain the power function $D=D_{0} u^{2((r+s-1) / s)}$.

Remark 3. By putting $r=1$, the corresponding system of class (1) admits still a 3-dimensional symmetry Lie algebra generated by translations in time and in space and by $X_{3}$. These results are in agreement with the ones obtained in [2].

By considering $s+r-1=0$ we do not get extension of $L_{\mathscr{P}}$ for $D$ and $F$ arbitrary. We get, instead, the following extension:

$$
X_{3}=s t \partial_{t}+x \partial_{x}+\Gamma_{1} s t u \partial_{u}+\frac{\Gamma_{1} s t}{b} v \partial_{v}
$$

provided that $D=D_{0}=$ const. and $F=\left(\Gamma_{1} / b\right) v$.

(2) Consider $\lambda(t, v)=\delta_{1}(t)=0$. In this case the only symmetries admitted are the translations in space and time and the form of $D$ and $F$ is arbitrary.
5.2. $s=2$. We analyze this case by beginning with the discussion of (56) from which two cases arise.

(1) $D(u)$ Is Arbitrary. It follows, of course, that $\delta=0$ and $\beta^{\prime}=$ $2 \alpha_{1}$ so

$$
\beta=2 \alpha_{1} t+\beta_{0}
$$

with $\beta_{0}$ arbitrary constant.

Moreover (46) and (48) become

$$
\begin{gathered}
-2 \alpha_{1} G+\alpha_{1} u_{x} G_{u_{x}}-\lambda(v) G_{v}=0, \\
\left(\lambda_{v}-2 \alpha_{1}\right) F-\lambda(v) F_{v}=0 .
\end{gathered}
$$

But taking into account that

$$
G=\rho u^{r} u_{x}^{2}+\Gamma_{1} u^{a}+\Gamma_{2} v^{b}
$$

(79) becomes

$$
-2 \alpha_{1}\left(\rho u^{r} u_{x}^{2}+\Gamma_{1} u^{a}+\Gamma_{2} v^{b}\right)+2 \alpha_{1} \rho u^{r} u_{x}^{2}-\lambda(v) b \Gamma_{2} v^{b-1}=0
$$

and gives us the following conditions:

$$
2 \alpha_{1} \Gamma_{1}=0, \quad 2 \alpha_{1} v+\lambda(v) b=0
$$

from where, taking into account the work hypotheses at the beginning of this section, we get $\alpha_{1}=\lambda=0$ with $F$ arbitrary function of $u$ and $v$.

In this case the only admitted symmetries are translations in time and space.

(2) Consider $D_{u} / D=\left(2 \alpha_{1}-\beta^{\prime}\right) / \delta(t, u)$. Then by requiring

$$
\frac{\partial}{\partial t}\left(\frac{2 \alpha_{1}-\beta^{\prime}}{\delta(t, u)}\right)=0,
$$

we get

$$
-\delta(t, u) \beta^{\prime \prime}-\left(2 \alpha_{1}-\beta^{\prime}\right) \delta_{t}=0
$$

from where we derive then

(a) $\delta=\delta(t, u)$ arbitrary function and $2 \alpha_{1}-\beta^{\prime}=0$ and

$$
\beta=2 \alpha_{1} t+\beta_{0}
$$

and $D_{u}=0$, so we omit this case,

(b)

$$
\delta(t, u)=\left(2 \alpha_{1}-\beta^{\prime}\right) A(u) .
$$

In this case

$$
\frac{D_{u}}{D}=\frac{1}{A(u)}
$$

which implies

$$
D(u)=D_{0} e^{\int d u / A(u)} \equiv D_{0} e^{a(u)},
$$


where

$$
a^{\prime}(u)=\frac{1}{A(u)} .
$$

Equation (47) after (54), (58), and (81) becomes

$$
\begin{aligned}
& -\beta^{\prime \prime} A(u)+\left(\left(2 \alpha_{1}-\beta^{\prime}\right) A^{\prime}(u)-\beta_{t}\right)\left(\rho u^{r} u_{x}^{2}+\Gamma_{1} u^{a}+\Gamma_{2} v^{b}\right) \\
& -\left(\delta_{u u} u_{x}^{2}\right) D_{0} e^{\int d u / A(u)}+ \\
& -\left(\left(2 \alpha_{1}-\beta^{\prime}\right) A^{\prime}(u)-\alpha_{1}\right) u_{x} 2 \rho u^{r} u_{x} \\
& -\left(2 \alpha_{1}-\beta^{\prime}\right) A(u)\left(r \rho u^{r-1} u_{x}^{2}+a \Gamma_{1} u^{a-1}\right) \\
& -\lambda(t, v) b \Gamma_{2} v^{b-1}=0 .
\end{aligned}
$$

From terms in $v$ we get the following condition:

$$
\left(\left(2 \alpha_{1}-\beta^{\prime}\right) A^{\prime}(u)-\beta^{\prime}\right) v-\lambda(t, v) b=0,
$$

which gives us $A^{\prime \prime}(u)=0$ and then $A=A_{1} u+A_{0}$. Therefore, from (87)

$$
\delta(t, u)=\left(2 \alpha_{1}-\beta^{\prime}\right)\left(A_{1} u+A_{0}\right),
$$

which implies that

$$
\delta_{u u}=0 .
$$

From (92) arise two cases.

(i) Consider $\lambda(t, v)=0$ and $\left(2 \alpha_{1}-\beta^{\prime}\right) A_{1}-\beta^{\prime}=0$. In this case after having derived from (91) the following additional conditions

$$
A_{0}=0, \quad \beta^{\prime \prime} A_{1}=0,
$$

$2 A_{1}\left(2 \alpha_{1}-\beta^{\prime}\right)(2-r)-2 \alpha_{1}=0, \quad A_{1}\left(2 \alpha_{1}-\beta^{\prime}\right) a \Gamma_{1}=0$,

it is a simple matter to ascertain that there does not exist extension of $L_{\mathscr{P}}$.

(ii) Consider $\lambda(t, v)=\lambda_{0}(t) v$ with

$$
\lambda_{0}(t)=\frac{1}{b}\left(\left(2 \alpha_{1}-\beta^{\prime}\right) A_{1}-\beta^{\prime}\right) .
$$

Then (91) assumes the following form:

$$
\begin{aligned}
& -\beta^{\prime \prime}\left(A_{1} u+A_{0}\right)+\left(\left(2 \alpha_{1}-\beta^{\prime}\right) A_{1}-\beta_{t}\right)\left(\rho u^{r} u_{x}^{2}+\Gamma_{1} u^{a}\right)+ \\
& -\left(\left(2 \alpha_{1}-\beta^{\prime}\right) A_{1}-\alpha_{1}\right) u_{x} 2 \rho u^{r} u_{x} \\
& -\left(2 \alpha_{1}-\beta^{\prime}\right)\left(A_{1} u+A_{0}\right)\left(r \rho u^{r-1} u_{x}^{2}+a \Gamma_{1} u^{a-1}\right)=0 .
\end{aligned}
$$

For $r \neq 1$, from (97) we obtain the following:

$$
\begin{gathered}
A_{0}=0, \quad \beta=\beta_{1} t+\beta_{0}, \\
\left(2 \alpha_{1}-\beta_{1}\right)\left(1-A_{1}(1+r)\right)=0, \\
\Gamma_{1}\left[\left(2 \alpha_{1}-\beta_{1}\right) A_{1}(1-a)-\beta_{1}\right]=0 .
\end{gathered}
$$

From the previous conditions we consider the following subclasses.

(A) Consider $2 \alpha_{1}-\beta_{1}=0$ and $\left(2 \alpha_{1}-\beta_{1}\right) A_{1}(1-a)-\beta_{1}=0$. As a consequence we get

$$
\beta_{1}=\alpha_{1}=\delta=\lambda=0,
$$

and then there is no extension of $L_{\mathscr{P}}$.

(B) For $1-A_{1}(1+r)=0$ and $\left(2 \alpha_{1}-\beta_{1}\right) A_{1}(1-a)-\beta_{1}=0$ we get, for $a \neq 1$,

$$
\begin{gathered}
A_{1}=\frac{1}{1+r}, \quad \alpha=\frac{\beta_{1}}{2}(2-a-r) x+\alpha_{0}, \quad \beta=\beta_{1} t+\beta_{0}, \\
\delta=\frac{\beta_{1}}{1-a} u, \quad \lambda=\beta_{1} \frac{1}{b} \frac{a}{1-a} v
\end{gathered}
$$

and then in this subcase we got an extension by one of $L_{\mathscr{P}}$ given by

$$
X_{3}=t \partial_{t}+\frac{2-a-r}{2} x \partial_{x}+\frac{u}{1-a} \partial_{t} u+\frac{a v}{(1-a) b} \partial_{v}
$$

provided that $D(v)$ and $F(u, v)$ are solutions of the following equations:

$$
\begin{gathered}
\frac{u}{1-a} D_{u}=(1-a-r) D, \\
(a-b(1-a)) F-b u F_{u}-a v F_{v}=0 .
\end{gathered}
$$

For $a=1$, instead, we conclude that

$$
\beta_{1}=0, \quad \delta=\frac{2 \alpha_{1}}{1+r}, \quad \lambda=\frac{1}{b} \frac{2 \alpha_{1}}{1+r} \nu .
$$

Therefore, the extension is given by

$$
X_{3}=x \partial_{x}+\frac{2}{1+r} u \partial_{u}+\frac{1}{b} \frac{2}{1+r} v
$$

provided that $D(u)$ and $F(u, v)$ are solutions of the following differential equations:

$$
\begin{gathered}
\frac{u}{1+r} D_{u}=D, \\
F-b u F_{u}-v F_{v}=0 .
\end{gathered}
$$

\section{Conclusions}

In this paper we have considered a class of reaction-diffusion systems with an additional advection term. The studied class includes, as particular cases, all partial differential equation models concerned with the Aedes aegypti mosquito that have been proposed until now. We have investigated such a system from the point of view of equivalence transformations in the spirit of the Lie-Ovsiannikov algorithm based on the Lie infinitesimal criterion. In agreement with some modifications of the Lie-Ovsiannikov algorithm, introduced in [20], we obtained a group of weak equivalence transformations. We 
have applied these transformations in order to obtain symmetry generators by using a projection theorem. In particular after having obtained the principal Lie algebra, we investigated a specific, but quite general, form for the advectionreaction term $G$ and we derived some extensions of the principal Lie algebra. The specializations of the results for the systems studied in [2] are in agreement with those ones obtained there.

\section{Conflict of Interests}

The authors declare that there is no conflict of interests regarding the publication of this paper.

\section{Acknowledgments}

The authors would like to thank FAPESP for financial support (Grants 2011/20072-0 and 2011/19089-6). Mariano Torrisi would also like to thank CMCC-UFABC for its warm hospitality and GNFM (Gruppo Nazionale per Fisica-Matematica) for its support. He also thanks the support from the University of Catania through PRA.

\section{References}

[1] L. T. Takahashi, N. A. Maidana, W. Castro Ferreira, Jr., P. Pulino, and H. M. Yang, "Mathematical models for the Aedes aegypti dispersal dynamics: travelling waves by wing and wind," Bulletin of Mathematical Biology, vol. 67, no. 3, pp. 509-528, 2005.

[2] I. L. Freire and M. Torrisi, "Symmetry methods in mathematical modeling of Aedes aegypti dispersal dynamics," Nonlinear Analysis. Real World Applications, vol. 14, no. 3, pp. 1300-1307, 2013.

[3] I. L. Freire and M. Torrisi, "Transformações de equivalência fracas para uma classe de modelos em biomatemática," in Anais do Congresso de Matemática Aplicada e Computacional (CMACCO '13), 2013.

[4] I. L. Freire and M. Torrisi, "Similarity solutions for systems arising from an Aedes aegypti model," Communications in Nonlinear Science and Numerical Simulation, vol. 19, no. 4, pp. 872-879, 2014.

[5] N. H. Ibragimov, M. Torrisi, and A. Valenti, "Preliminary group classification of equations $v_{t t}=f\left(x, v_{x}\right) v_{x x}+g\left(x, v_{x}\right)$," Journal of Mathematical Physics, vol. 32, no. 11, pp. 2988-2995, 1991.

[6] I. Lisle, Equivalence transformations for classes of differential equations [Ph.D. thesis], University of British Columbia, 1992.

[7] M. Senthilvelan, M. Torrisi, and A. Valenti, "Equivalence transformations and differential invariants of a generalized nonlinear Schrödinger equation," Journal of Physics A, vol. 39, no. 14, pp. 3703-3713, 2006.

[8] M. L. Gandarias, M. Torrisi, and R. Tracinà, "On some differential invariants for a family of diffusion equations," Journal of Physics A, vol. 40, no. 30, pp. 8803-8813, 2007.

[9] J.-P. Gazeau and P. Winternitz, "Symmetries of variable coefficient Korteweg-de Vries equations," Journal of Mathematical Physics, vol. 33, no. 12, pp. 4087-4102, 1992.

[10] P. Winternitz and J.-P. Gazeau, "Allowed transformations and symmetry classes of variable coefficient Korteweg-de Vries equations," Physics Letters A, vol. 167, no. 3, pp. 246-250, 1992.
[11] L. V. Ovsiannikov, Group Analysis of Differential Equations, Academic Press, New York, NY, USA, 1982.

[12] I. S. Akhatov, R. K. Gazizov, and N. K. Ibragimov, "Nonlocal symmetries. Heuristic approach," Journal of Soviet Mathematics, vol. 55, no. 1, pp. 1401-1450, 1991.

[13] M. Torrisi and R. Tracinà, "Equivalence transformations and symmetries for a heat conduction model," International Journal of Non-Linear Mechanics, vol. 33, no. 3, pp. 473-487, 1998.

[14] V. Romano and M. Torrisi, "Application of weak equivalence transformations to a group analysis of a drift-diffusion model," Journal of Physics A, vol. 32, no. 45, pp. 7953-7963, 1999.

[15] N. H. Ibragimov, CRC Handbook of Lie Group Analysis of Differential Equations, CRC Press, Boca Raton, Fla, USA, 1996.

[16] M. Molati and C. M. Khalique, "Lie group classification of a generalized Lane-Emden type system in two dimensions," Journal of Applied Mathematics, vol. 2012, Article ID 405978, 10 pages, 2012.

[17] C. M. Khalique, F. M. Mahomed, and B. P. Ntsime, "Group classification of the generalized Emden-Fowler-type equation," Nonlinear Analysis. Real World Applications, vol. 10, no. 6, pp. 3387-3395, 2009.

[18] M. Torrisi and R. Tracina, "Equivalence transformations for systems of first order quasilinear partial differential equations," in Modern Group Analysis VI: Developments in Theory, Computation and Application, N. H. Ibragimov and F. M. Mahomed, Eds., New Age International, 1996.

[19] M. Torrisi, R. Tracinà, and A. Valenti, "A group analysis approach for a nonlinear differential system arising in diffusion phenomena," Journal of Mathematical Physics, vol. 37, no. 9, pp. 4758-4767, 1996.

[20] M. Torrisi and R. Tracinà, "Exact solutions of a reactiondiffusion systems for Proteus mirabilis bacterial colonies," Nonlinear Analysis. Real World Applications, vol. 12, no. 3, pp. 18651874, 2011.

[21] G. Gambino, A. M. Greco, and M. C. Lombardo, "A group analysis via weak equivalence transformations for a model of tumour encapsulation," Journal of Physics A, vol. 37, no. 12, pp. 3835-3846, 2004.

[22] N. H. Ibragimov and N. Säfström, "The equivalence group and invariant solutions of a tumour growth model," Communications in Nonlinear Science and Numerical Simulation, vol. 9, no. 1, pp. 61-68, 2004.

[23] M. Torrisi and R. Tracinà, "Second-order differential invariants of a family of diffusion equations," Journal of Physics A, vol. 38, no. 34, pp. 7519-7526, 2005.

[24] M. Torrisi and R. Tracinà, "On a class ofd reaction diffusion systems: E quivalence transformations and symmetries," in Asymptotic Methods in Nonlinear Wave Phenomena, T. Ruggeri and M. Sammartino, Eds., pp. 207-216, 2007.

[25] N. H. Ibragimov and M. Torrisi, "A simple method for group analysis and its application to a model of detonation," Journal of Mathematical Physics, vol. 33, no. 11, pp. 3931-3937, 1992. 


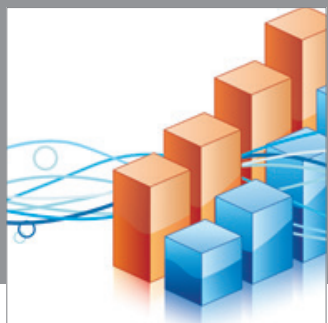

Advances in

Operations Research

mansans

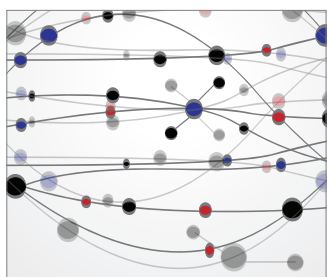

The Scientific World Journal
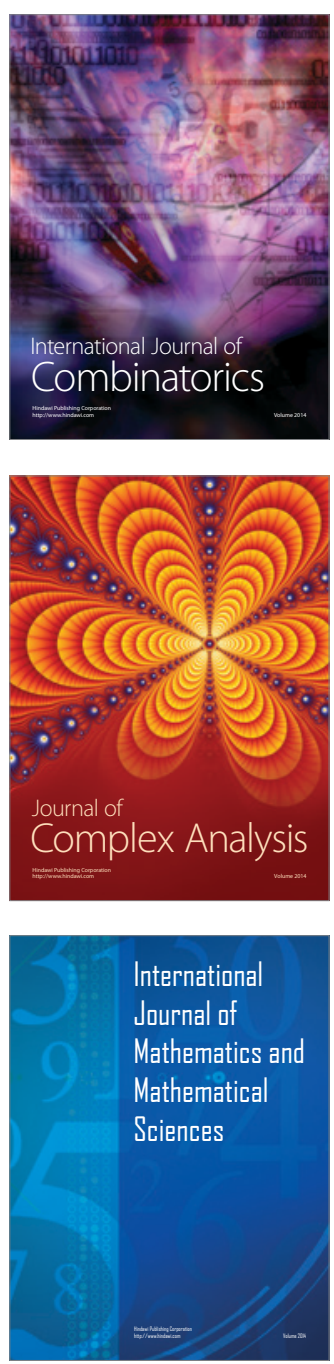
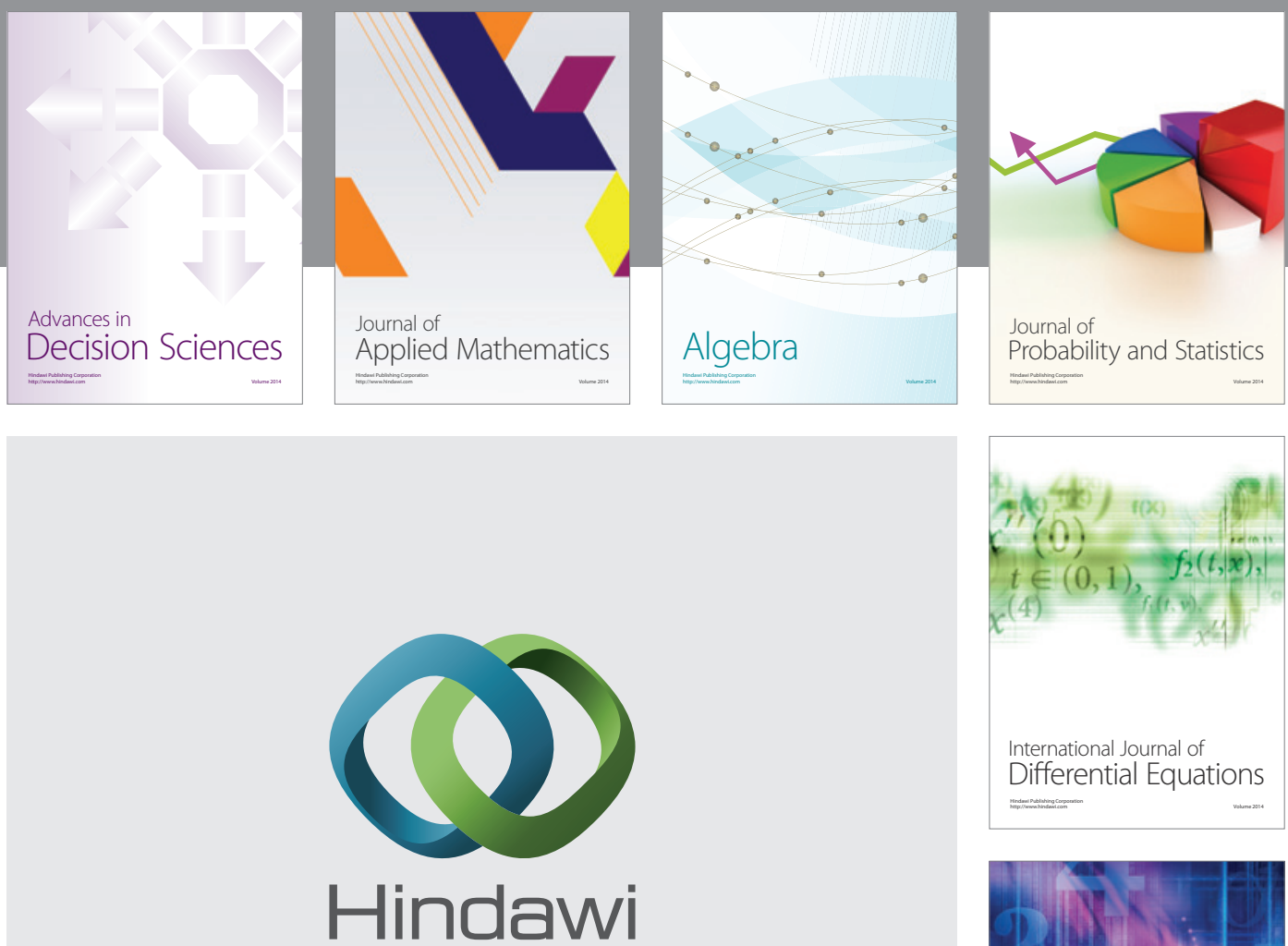

Submit your manuscripts at http://www.hindawi.com
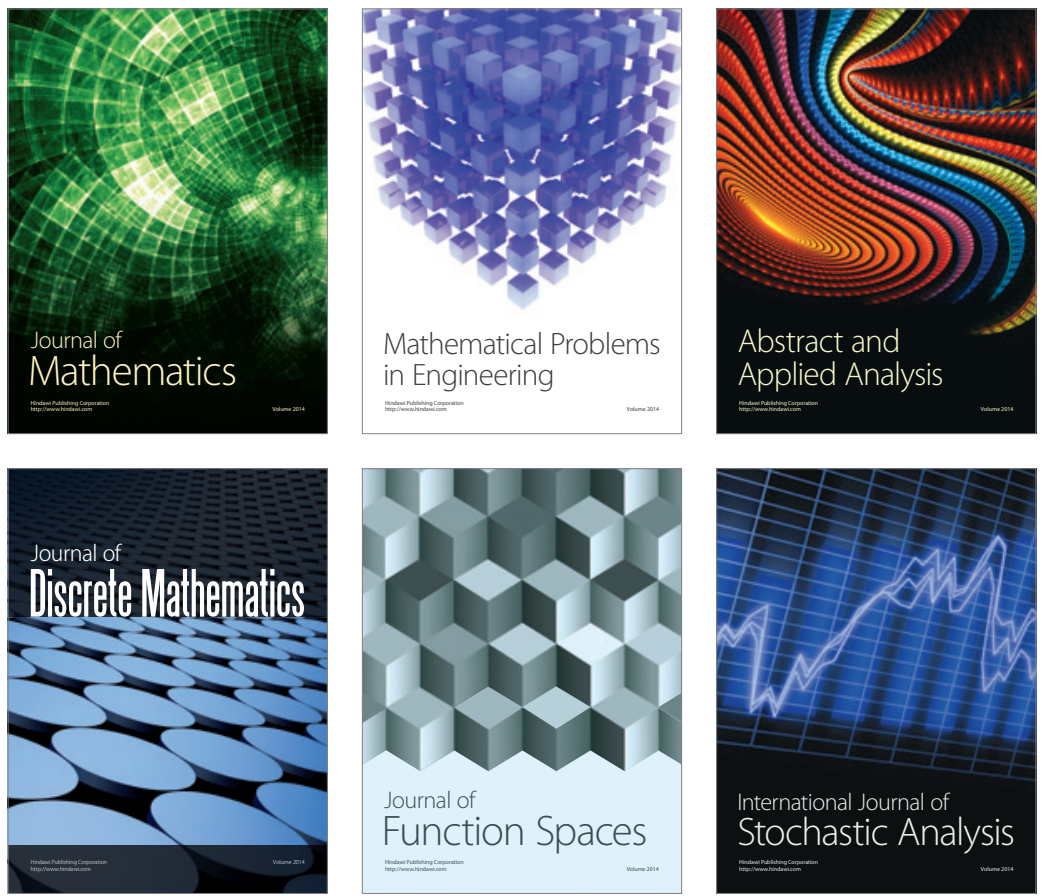

Journal of

Function Spaces

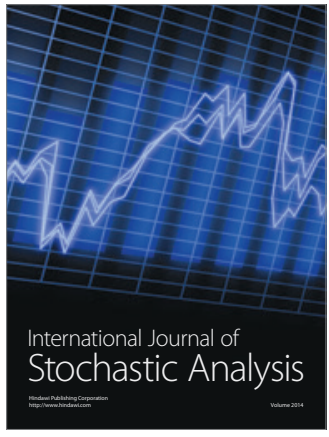

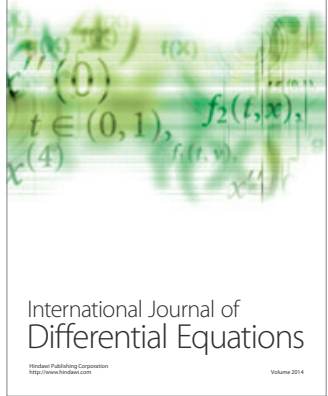
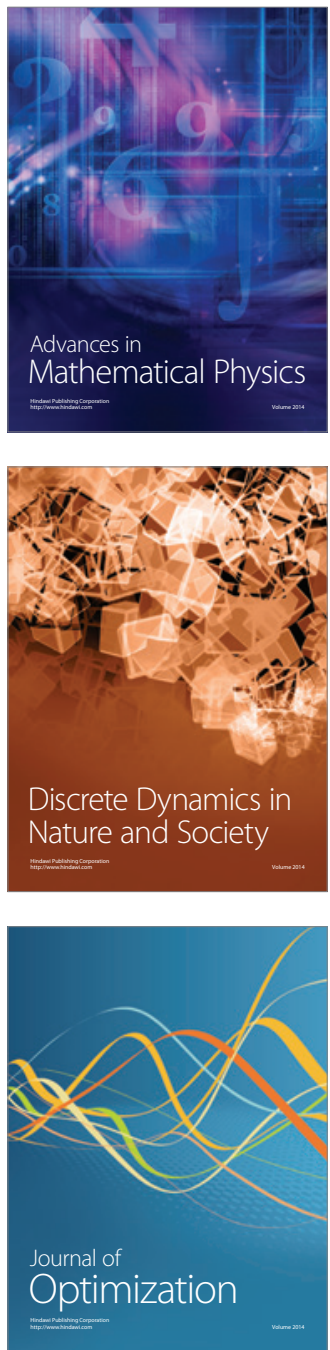